\title{
Localized eigenmodes of the covariant lattice Laplacian $^{\dagger}$
}

\section{J. Greensite*}

The Niels Bohr Institute, Blegdamsvej 17, DK-2100 Copenhagen Ø, Denmark;

Physics and Astronomy Dept., San Francisco State University, San Francisco, CA 94117, USA

E-mail: greensitestars.sfsu.edu

\section{Š. Olejník}

Institute of Physics, Slovak Academy of Sciences, SK-845 11 Bratislava, Slovakia

E-mail: stefan.olejnikesavba.sk

\section{I. Polikarpov, S. N. Syritsyn}

ITEP, B. Cheremushkinskaya 25, Moscow 117259, Russia

E-mail: polykarpeitep.ru, syritsyneitep.ru

\section{I. Zakharov}

Max-Planck Institüt für Physik, Föhringer Ring 6, D-80805 Munich, Germany

E-mail: xxz@mppmu.mpg.de

We study numerically the eigenmode spectrum of the covariant lattice Laplacian, in the fundamental SU(2) color group representation. It is found that eigenmodes at the lower and upper ends of the spectrum are localized, and that the localization volume scales. In contrast, the eigenmodes of the lattice Faddeev-Popov operator are all extended rather than localized (as required for confinement) despite the similarity of the kinetic and Faddeev-Popov operators.

XXIIIrd International Symposium on Lattice Field Theory

25-30 July 2005

Trinity College, Dublin, Ireland

\footnotetext{
$\dagger$ Our research is supported in part by the U.S. Department of Energy under Grant No. DE-FG03-92ER40711 (J.G.), the Slovak Science and Technology Assistance Agency under Contract No. APVT-51-005704 (Š.O.), grants RFBR 0402-16079, RFBR 05-02-16306-a (M.I.P.), grants RFBR 05-02-16306-a, RFBR 05-02-17642, and an Euler-Stipendium (S.N.S.).

* Speaker.
} 


\section{Introduction}

It is well-known from condensed-matter physics that electron propagation in a periodic potential is described by Bloch waves, which are extended, plane-wave-like states. However, when disorder is introduced into the potential, low-lying electron eigenstates become exponentially localized, as shown long ago by Anderson. This is an interference effect due to multiple scattering, rather than ordinary bound state formation in a single potential well. When the energy of the highest localized state (the "mobility edge") exceeds the Fermi energy, the material is an insulator.

Recently, localization in the lowest eigenmodes of the lattice Dirac operator has been intensely studied, in the hope that it might shed light on properties and dimensionality of important underlying structures in the QCD vacuum. It was found that:

- Wilson-Dirac fermions have a low-lying spectrum of localized eigenmodes in certain regions of the phase diagram [1];

- low-lying modes of the Asqtad fermion operator, although extended, seem to concentrate on lattice sub-volumes of dimensionality < 4 [2];

- low-lying modes of the overlap Dirac operator are localized on volumes which shrink as a power of the lattice spacing $a$ [3].

Some questions naturally arise: If fermionic operators are picking up signals of lower-dimensional substructure, is there any relation to, e.g., center vortex sheets or monopole worldlines? Can one find indications of lattice-scale 2-brane structures, along the lines suggested by Zakharov 㺻?

In the present study we concentrate on the following questions:

- Is localization/concentration unique to Dirac operator eigenmodes, or is it found in other lattice kinetic operators, e.g. the Faddeev-Popov and covariant Laplacian operators? ${ }^{1}$

- If so, is there any connection to confinement?

Here we sketch a subset of our results, other can be found in [5, 6].

\section{Signals of localization}

The covariant lattice Laplacian in the $j$-th representation of the $\mathrm{SU}(2)$ gauge group is

$$
\triangle_{x y}^{a b}=\sum_{\mu}\left[U_{\mu}^{a b}(x) \delta_{y, x+\hat{\mu}}+U_{\mu}^{\dagger a b}(x-\hat{\mu}) \delta_{y, x-\hat{\mu}}-2 \delta^{a b} \delta_{x y}\right]
$$

(color indices $a, b$ run from 1 to $2 j+1$ ) and we are interested in the low-lying eigenmodes $\phi_{n}^{a}(x)$ satisfying the eigenvalue equation $-\triangle_{x y}^{a b} \phi_{n}^{b}(y)=\lambda_{n} \phi_{n}^{a}(x)$.

As probes of localization, we use two quantities:

- The Inverse Participation Ratio (IPR) of the $n$-th eigenmode, defined by

$$
I P R_{n}=V\left\langle\sum_{x} \rho_{n}^{2}(x)\right\rangle, \quad \text { where } \quad \rho_{n}(x)=\sum_{a}\left|\phi_{n}^{a}(x)\right|^{2}
$$

\footnotetext{
${ }^{1}$ Note that the covariant Laplacian is not the square of the Dirac operator. (This is only true for the free theory.) That means that the eigenmodes of the covariant Laplacian need not be directly related to the eigenmodes of the Dirac operator; they might have completely different localization properties.
} 
is the normalized eigenmode density. If the eigenmode is extended over the whole lattice, $\rho \approx 1 / V$, and $I P R=\mathscr{O}(1)$. In contrast, for an eigenmode localized in a volume $b$ (i.e. $\rho \approx 0$ except in a region of volume $b), I P R \approx 1 / b$.

- The Remaining Norm $(R N)$, defined in the following way: Sort $\rho(x)$ into a one-dimensional array $r(k), k=1,2, \ldots, V$, with $r(k) \geq r(k+1)$. Then the $R N$ is

$$
R N(K)=1-\sum_{k=1}^{K} r(k)
$$

The $R N$ is the amount of total norm (=1) remaining after counting contributions from the $K<V$ subset of sites with largest $\rho(x)$.

\section{Results for the fundamental $(j=1 / 2)$ representation}

Evidence of localization. We calculated the average $I P R$ of the lowest-lying eigenmode at various $\beta$ values, and fit the data at each coupling to $I P R=A+L^{4} / b$. Fig. 1 (left part) shows the data in a $\log -\log$ plot. It is quite clear that $(I P R-A)$ is proportional to the lattice volume, indicating that the eigenmode is localized in a 4-volume. In Fig. 1 (right) we plot $(I P R-A)$ vs the lattice volume in physical units, $V\left[\mathrm{fm}^{4}\right]=V a^{4}(\beta)=\left(L a^{4}(\beta)\right)$, where $a(\beta)$ denotes the lattice spacing (in $\mathrm{fm}$ ) at coupling $\beta$. The data fall roughly on a straight line, which implies that the localization 4-volume $\left(b a^{4}\right)$ is constant in physical units, about $(2.3 \mathrm{fm})^{4}$.

Locating/removing center vortices. The next question is whether the localization is due to some confining disorder of the lattice configuration. Therefore we computed the spectrum of the covariant Laplacian in the center-projected and vortex-removed configurations. We first fixed lattice configurations into the (direct) maximal center gauge (MCG) and obtained "vortex-only" configurations by center projection [7]. The "vortex-removed" configurations were then obtained by multiplying the lattice configuration in MCG by the center-projected one [8]. Vortex removal is quite a minimal change - only the action at $\mathrm{P}$-vortex plaquettes (plaquettes equal to -1 after center projection) is changed, and the density of those plaquettes drops exponentially with $\beta$. How is localization affected?
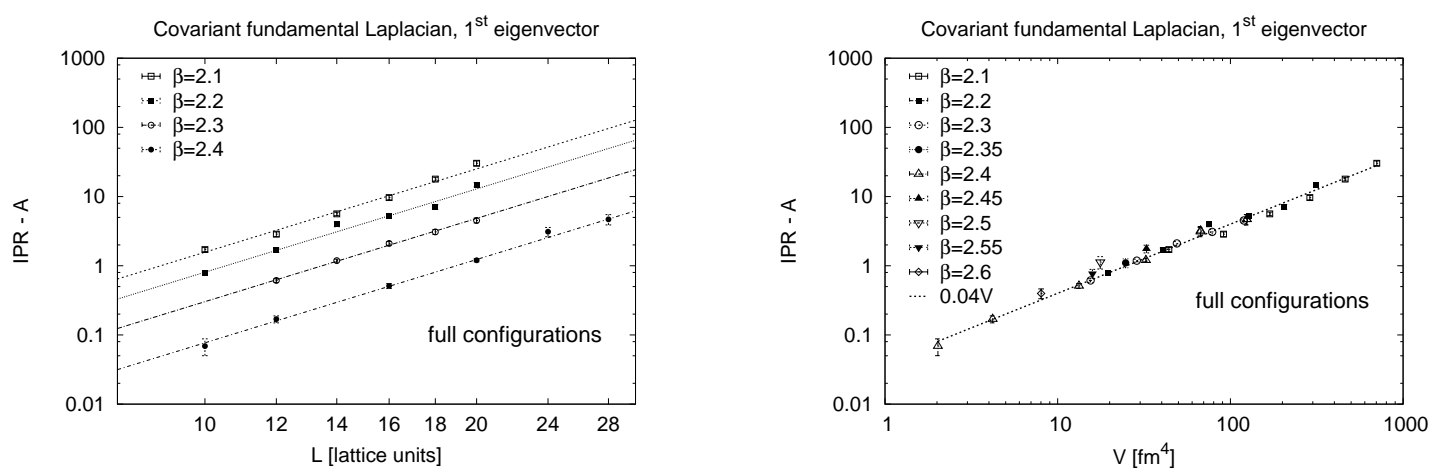

Figure 1: [Left] $\log -\log$ plot of $I P R-A$ vs lattice length $L$, at various $\beta$. The lines show a fit to $I P R=$ $A+L^{4} / b$. [Right] Log-log plot of $I P R-A$ vs physical volume $V=(L a)^{4}$. 

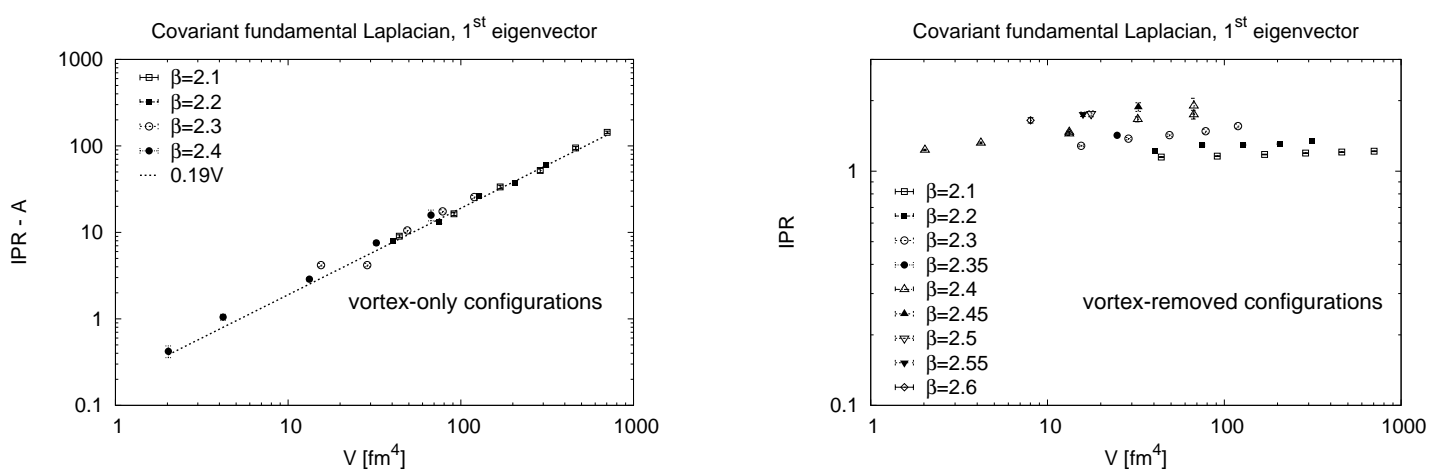

Figure 2: Log-log plot of $I P R-A$ vs physical volume in vortex-only configurations [left] and vortexremoved configurations [right].

Results are shown in Fig. 2. We find a somewhat greater localization (left part) in vortex-only configurations. $b_{\text {phys }}$ is reduced in this case to about $(1.5 \mathrm{fm})^{4}$. The situation drastically changes in vortex-removed configurations, the eigenmodes turn out to be extended (see Fig. 2, right).

The same conclusion follows from examining the Remaining Norm (see [5]): For the unmodified configurations, the $\mathrm{RN}$ curve becomes slightly broader with increasing lattice volume, but the $R N$ data seem to converge to a limiting curve at the largest volumes. The convergence is clearer in vortex-only configurations, where almost all data fall on essentially the same curve. For the vortex-removed data, the curve broadens as the volume increases; again, this means no localization without center vortices.

Mobility edges. Not all eigenmodes of the covariant Laplacian in the fundamental representation are localized, only the low- and high-lying modes. ${ }^{2}$ The bulk of states are extended. This is shown in Fig. 目 for full and vortex-only configurations. One could estimate ("by the eye") the mobility edge at $\beta=2.1$ to be around $\lambda=1.45$ in the former, and about 1.15 in the latter case.
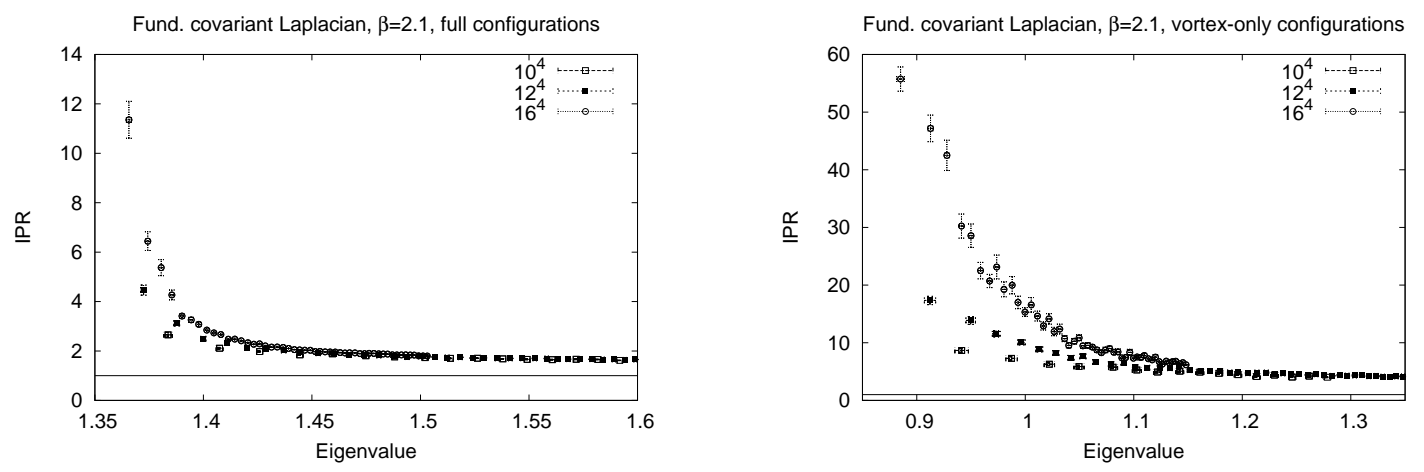

Figure 3: IPRs of the first 100 eigenmodes in full [left] and vortex-only configurations [right].

\footnotetext{
${ }^{2}$ If $\phi(x)$ is an eigenmode, so is $\phi^{\prime}(x)=(-1)^{\sum_{\mu} x_{\mu}} \phi(x)$. One can then show that $I P R_{n}=I P R_{n_{\max }-n+1}$. Localization at the lower end of the spectrum implies localization at the upper end [5].
} 


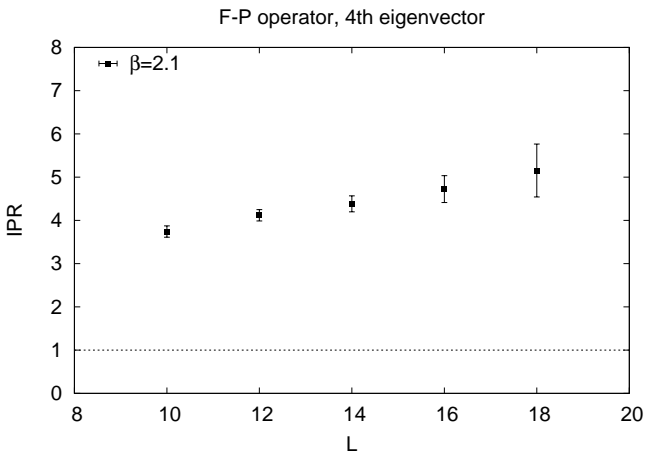

Figure 4: IPR of the lowest nontrivial eigenmode of the FP operator.

\section{The Faddeev-Popov Operator}

We have argued that localization of the low-lying states of scalar particles implies a mass gap, i.e. the bare scalar mass cannot be adjusted to zero and scalar field correlators cannot be long-range (see [5]). A check of the logic of the argument is provided by the Faddeev-Popov (FP) operator in Coulomb gauge. On the one hand, it looks similar to the covariant Laplacian (in $D=3$ dimensions): $\mathscr{M}=-\vec{\nabla} \cdot \vec{D}(A)[\mathscr{D}(A)$ is the covariant derivative]. One might therefore expect that the low-lying eigenvalues are localized. On the other hand, the Coulomb energy of a given charge distribution is $H_{\text {coul }}=\frac{1}{2} \int d^{3} x d^{3} y \rho^{a}(x) K^{a b}(x, y ; A) \rho^{b}(y), \quad$ where $\quad K^{a b}(x, y ; A)=\left[\mathscr{M}^{-1}\left(-\nabla^{2}\right) \mathscr{M}^{-1}\right]_{x y}^{a b}$, so if $\mathscr{M}^{-1}$ is short-range, the Coulomb potential $\sim K(x, y)$ is short range as well.

But in fact, we know that asymptotically [9] $\langle K(x, y, A)\rangle \sim \sigma_{\text {coul }}|x-y|$, where $\sigma_{\text {coul }} \approx 3 \sigma$, so it must be that the low-lying eigenmodes of the FP operator are not localized. The IPR's for the lowest nontrivial eigenmode of the FP operator at $\beta=2.1$ are shown in Fig. 4 . Despite the similarity to the covariant Laplacian, we see no apparent localization - in agreement with GribovZwanziger Coulomb-gauge confinement scenario [10] and the claim formulated at the beginning of this section.

\section{What feature of Laplacian eigenmodes is crucial to confinement?}

If localization is not crucial to confinement, then what feature is? Let us suppose we have a dynamical scalar field $\phi(x)$ in the Lagrangian. Consider the Euclidean propagator in Coulomb gauge $D(t)=\left\langle\phi^{\dagger a}(t) \phi(0)\right\rangle=\sum_{k} c_{k} e^{-E_{k} t}$, where the $E_{k}$ are the excitation energies of (non-singlet) states with the quantum numbers of the scalar. These should be infinite, in a confining theory. Then, in the quenched approximation,

$$
D(t)=\sum_{n}\left\langle\frac{\phi_{n}^{\dagger a}(t) \phi_{n}^{a}(0)}{\lambda_{n}+m_{0}^{2}}\right\rangle=0, \quad \text { where } m_{0}^{2} \text { is the bare mass. }
$$

The natural implication is that $G_{n}(t) \equiv\left\langle\phi_{n}^{\dagger a}(t) \phi_{n}^{a}(0)\right\rangle=0$ for any eigenmode, localized or extended, and any time difference $t>0$. 
This we indeed find numerically in the confining phase, i.e. $G_{n}(0, t) \approx 0, G_{n}(\vec{x}, 0) \neq 0$. When center vortices are removed, or in the Higgs phase, then also $G_{n}(0, t) \neq 0$. This is a consequence of spontaneous breaking of the remnant global gauge symmetry that exists in Coulomb gauge [11].

\section{Conclusions}

In the fundamental $(j=1 / 2)$ representation of $\mathrm{SU}(2)$ we find that the low lying eigenmodes of the covariant Laplacian operator are localized, and that

- $b a^{4}$ is $\beta$-independent: localization volume is fixed in physical units;

- the same is true for "vortex-only" configurations; localization volume is smaller;

- localization disappears when vortices are removed;

- there are no localized eigenmodes for the Faddeev-Popov operator, as required in Coulombgauge confinement scenarios [10].

One might suppose that the situation will be much the same for higher group representations. In fact there are some surprises, which were discussed in Sergey Syritsyn's talk [6].

\section{References}

[1] M. Golterman and Y. Shamir, Localization in lattice QCD, Phys. Rev. D 68 (2003) 074501 [hep-lat/0306002].

[2] C. Aubin et al., The scaling dimension of low lying Dirac eigenmodes and of the topological charge density, Nucl. Phys. Proc. Suppl. 140 (2005) 626 [hep-lat / 0410024 ]; see also Jim Hetrick's talk at this conference, PoS(LAT2005)299.

[3] F. V. Gubarev, S. M. Morozov, M. I. Polikarpov, and V. I. Zakharov, Low lying eigenmodes localization for chirally symmetric Dirac operator, hep-lat/0505016.

[4] V. I. Zakharov, Lower-dimension vacuum defects in lattice Yang-Mills theory, Phys. Atom. Nucl. 68 (2005) 573 [hep-ph/ 0410034$].$

[5] J. Greensite, Š. Olejník, M. Polikarpov, S. Syritsyn, and V. Zakharov, Localized eigenmodes of covariant Laplacians in the YM vacuum, Phys. Rev. D 71 (2005) 114507 [hep-lat/ 0504008 ].

[6] S. Syritsyn, Eigenmodes of covariant Laplacian in SU(2) Yang-Mills vacuum: higher representations, in proceedings of Lattice 2005, PoS(LAT2005)326.

[7] L. Del Debbio, M. Faber, J. Giedt, J. Greensite, and Š. Olejník, Detection of center vortices in the lattice Yang-Mills vacuum, Phys. Rev. D 58 (1998) 094501 [hep-lat/9801027].

[8] Ph. de Forcrand and M. D'Elia, Relevance of center vortices to QCD, Phys. Rev. Lett. 82 (1999) 4582 [hep-lat/9901020].

[9] J. Greensite and Š. Olejník, Coulomb energy, vortices, and confinement, Phys. Rev. D 67 (2003) 094503 [hep-lat/0302018].

[10] V. Gribov, Quantization of non-Abelian gauge theories, Nucl. Phys. B139 (1978) 1; D. Zwanziger, Renormalization in the Coulomb gauge and order parameter for confinement in QCD, Nucl. Phys. B518 (1998) 237.

[11] J. Greensite, Š. Olejník, and D. Zwanziger, Coulomb energy, remnant symmetry, and the phases of non-Abelian gauge theories, Phys. Rev. D 69 (2004) 074506 [hep-lat/ 0401003 ]. 